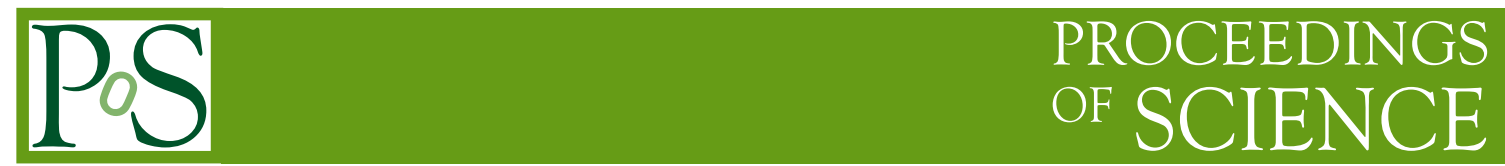

\title{
Non-standard heavy vectors at the LHC
}

\author{
Gennaro Corcella* \\ INFN, Laboratori Nazionali di Frascati \\ Via E. Fermi 40, 00044 Frascati (RM), Italy \\ E-mail: gennaro.corcella@lnf.infn.it
}

Heavy vector-boson hunting is one of the major analyses undertaken by the experiments carried out at the LHC. I will explore two scenarios which have not been investigated yet by the experimental collaborations. The first scenario consists in searching for $Z^{\prime}$ bosons, predicted by $\mathrm{U}(1)^{\prime}$ GUT-inspired models, by studying its supersymmetric decays, leading to resonant final states with charged leptons and missing energy. Afterwards, I shall discuss the 331 model and the production of doubly-charged vector bileptons, yielding events with two same-sign lepton pairs at the LHC.

The European Physical Society Conference on High Energy Physics

5-12 July, 2017

Venice

${ }^{*}$ Speaker. 


\section{Introduction}

Hunting for heavy vectors is one of the challenging goals of the LHC collaborations, since such vectors may represent a possible evidence of physics beyond the Standard Model. Heavy neutral gauge bosons $Z^{\prime}$ are predicted in extensions of the Standard Model (SM) based on U(1)' symmetries inspired by Grand Unification Theories (GUT) [1] and in the Sequential Standard Model (SSM), the simplest extension of the SM, wherein $Z^{\prime}$ and $W^{\prime}$ bosons have the same coupling to fermions as $Z$ and $W$. The LHC experiments have so far assumed that the $Z^{\prime}$ has only SM decay modes and have searched for high-mass dileptons $[2,3]$ and dijets $[4,5]$, setting exclusion limits on the $Z^{\prime}$ mass in the multi-TeV range.

In this talk, I explore possible supersymmetric decays of GUT-inspired $Z^{\prime}$ bosons, within the Minimal Supersymmetric Standard Model (MSSM), along the lines of Refs. [6, 7]. The opening of new channels lowers the SM branching ratios and the exclusion limits; moreover, if a $Z^{\prime}$ were to be discovered, its decays would be an ideal place to look for supersymmetry, since the $Z^{\prime}$ mass would set a constraint on sparticle invariant masses. In particular, decays into the lightest neutralinos are useful for the purpose of Dark Matter searches at colliders.

The other scenario which I shall tackle is the so-called 331 model, in the formulation of [8], which features five additional gauge bosons, four charged and one neutral, plus three exotic quarks. One of the main signatures of this model consists of the production of doubly-charged vector bileptons, decaying into same-sign charged-lepton pairs. ATLAS and CMS did explore doublycharged Higgs bosons $H^{ \pm \pm}[9,10]$, i.e. scalar bileptons, setting model-dependent exclusion bounds of several hundreds of $\mathrm{GeV}$, but never searched for vector bileptons. In this talk, I will investigate processes with two vector bileptons and two jets at the LHC, and compare their signal with SM backgrounds.

Section 2 will be devoted to $Z^{\prime}$ bosons within supersymmetry, discussing first the theoretical framework and then the phenomenology, while Section 3 will deal with the 331 model and bilepton production at the LHC. I will finally make some concluding remarks in Section 4.

\section{Supersymmetry and $Z^{\prime}$ bosons}

\subsection{Theoretical framework}

$\mathrm{U}(1)^{\prime}$ symmetries and $Z^{\prime}$ bosons typically arise from the breaking of a rank-6 GUT group $\mathrm{E}_{6}$ :

$$
\mathrm{E}_{6} \rightarrow \mathrm{SO}(10) \times \mathrm{U}(1)_{\psi}^{\prime} \rightarrow \mathrm{SU}(5) \times \mathrm{U}(1)_{\chi}^{\prime} \times \mathrm{U}(1)_{\psi}^{\prime}
$$

A generic $Z^{\prime}$ is given by the mixing between $Z_{\psi}^{\prime}$ and $Z_{\chi}^{\prime}$, associated with $\mathrm{U}(1)_{\psi}^{\prime}$ and $\mathrm{U}(1)_{\chi}^{\prime}$ :

$$
Z^{\prime}(\theta)=Z_{\psi}^{\prime} \cos \theta-Z_{\chi}^{\prime} \sin \theta
$$

This contribution will be focused on the $Z_{\psi}^{\prime}$ and its supersymmetric decays, since it is the framework yielding the largest cross section [7]. Scenarios with other values of $\theta$ were detailed in [6, 7].

In the MSSM, besides the scalar Higgs doublets $H_{d}$ and $H_{u}$, a singlet $S$ is necessary to break the $\mathrm{U}(1)^{\prime}$ symmetry and give mass to the $Z^{\prime}$. After electroweak symmetry breaking, the Higgs sector consists of one pseudoscalar $A$ and three scalars $h, H$ and a new $H^{\prime}$. In the gaugino sector, one will have two extra neutralinos, whereas the charginos are unchanged; furthermore, the $\mathrm{U}(1)^{\prime}$ group leads to extra D- and F-term corrections to sfermion masses. 

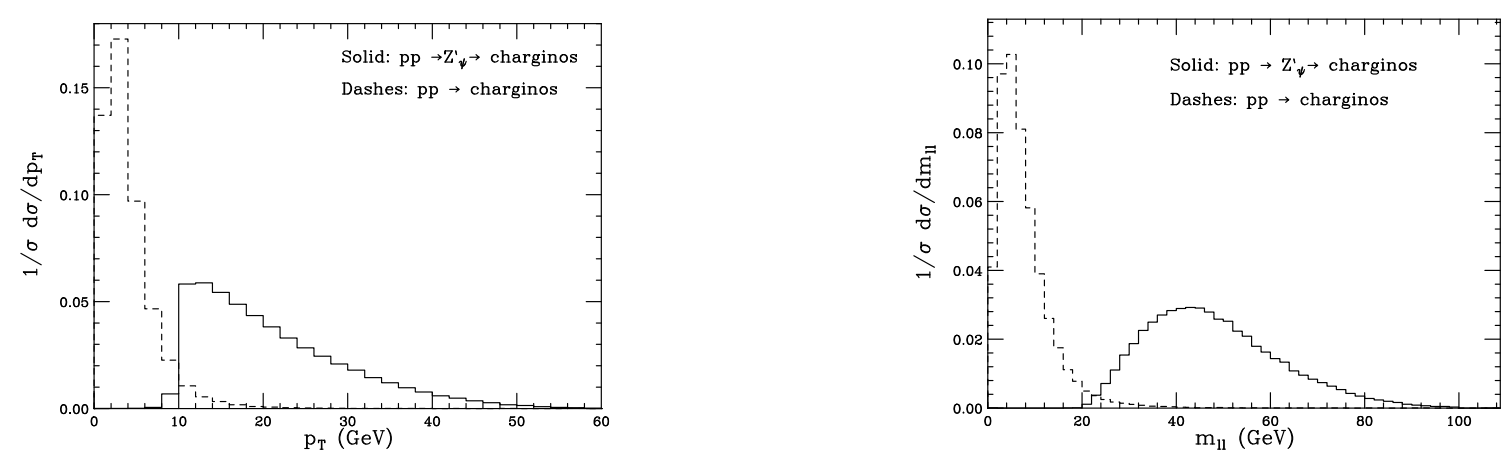

Figure 1: Left: lepton transverse momentum for the $Z_{\psi}^{\prime}$ model at $\sqrt{s}=14 \mathrm{TeV}$ in $Z_{\psi}^{\prime} \rightarrow \tilde{\chi}_{1}^{+} \chi_{1}^{-}$events and direct chargino-production processes. Right: invariant mass of the two charged leptons.

\subsection{Phenomenology of supersymmetric $Z^{\prime}$ decays}

Following [7], I shall discuss the phenomenology of a $Z_{\psi}^{\prime}$ boson with mass $m_{Z^{\prime}}=2 \mathrm{TeV}$ and require that the coupling constants of $\mathrm{U}(1)^{\prime}$ and $\mathrm{U}(1)_{\mathrm{Y}}$ are proportional via $g^{\prime}=\sqrt{5 / 3} g_{1}$. As for the MSSM parameters, I shall set: $M_{1}=400 \mathrm{GeV}, M^{\prime}=1 \mathrm{TeV}, \tan \beta=30, \mu=200 \mathrm{GeV}$ and $A_{q}=A_{\ell}=A_{\lambda}=4 \mathrm{TeV}$, where $A_{q}$ and $A_{\ell}$ are the soft trilinear couplings of squarks and sleptons with the Higgs fields and $A_{\lambda}$ is the soft Higgs trilinear coupling.

The sfermion spectrum, corresponding to soft masses at the $Z^{\prime}$ scale $m_{\tilde{\ell}}^{0}=m_{\tilde{v}_{\ell}}^{0}=1.2 \mathrm{TeV}$ and $m_{\tilde{q}}^{0}=5.5 \mathrm{TeV}$, computed by means of the SARAH [11] and SPheno [12] codes, is given in the tables reported in [7] and is omitted here for the sake of brevity. In the reference point of Ref. [7], the branching ratio into supersymmetric final states is almost 30\%: the highest rate is into chargino pairs $\tilde{\chi}_{1}^{+} \tilde{\chi}_{1}^{-}$, accounting for about $10 \%$, while the one into the lightest neutralinos, Dark Matter candidates, is roughly $5 \%$. An interesting supersymmetric channel can therefore be:

$$
p p \rightarrow Z_{\psi}^{\prime} \rightarrow \tilde{\chi}_{1}^{+} \tilde{\chi}_{1}^{-} \rightarrow\left(\tilde{\chi}_{1}^{0} \ell^{+} v_{\ell}\right)\left(\tilde{\chi}_{1}^{0} \ell^{-} \bar{v}_{\ell}\right)
$$

with $\ell=\mu, e$, leading to two charged leptons and missing energy. Its cross section, calculated at leading order (LO) by MadGraph [13], is $7.9 \times 10^{-4} \mathrm{pb}$ at $14 \mathrm{TeV}$.

Figure 1 (left) presents the transverse-momentum spectrum of the leptons produced in Eq. (2.3), according to MadGraph+HERWIG [14], as well as in processes with direct chargino production, i.e. $p p \rightarrow \tilde{\chi}_{1}^{+} \tilde{\chi}_{1}^{-}$, followed by $\tilde{\chi}^{+} \rightarrow \tilde{\chi}_{1}^{0} \ell^{+} v_{\ell}$. For direct chargino production, the leptons are rather soft and the spectrum is peaked at low $p_{T}$; as for $Z^{\prime}$-driven charginos, the $\tilde{\chi}_{1}^{+} \tilde{\chi}_{1}^{-}$invariant mass must be equal to $m_{Z^{\prime}}$, and therefore the lepton- $p_{T}$ distribution is substantial at higher $p_{T}$ and can be easily discriminated. The right panel of Fig. 1 displays the $\ell^{+} \ell^{-}$invariant-mass distribution: for $Z^{\prime}$ decays into charginos, $m_{\ell \ell}$ lies in the range $20 \mathrm{GeV}<m_{\ell \ell}<100 \mathrm{GeV}$ and is maximum at $m_{\ell \ell} \simeq 45 \mathrm{GeV}$; for direct $\tilde{\chi}_{1}^{+} \tilde{\chi}_{1}^{-}$production, $m_{\ell \ell}$ is peaked around $5 \mathrm{GeV}$ and rapidly vanishes. Decays into neutralino pairs $Z^{\prime} \rightarrow \tilde{\chi}_{1}^{0} \tilde{\chi}_{1}^{0}$ are relevant for the searches for Dark Matter candidates: it was shown in [7] that, although the shape of the missing-energy spectrum is similar to the one due to decays into neutrinos, the overall production cross section of invisible particles is remarkably increased, once accounting for neutralinos. As a whole, even though a specific analysis on supersymmetric $Z^{\prime}$ models is currently missing, using the LHC data at $8 \mathrm{TeV}$ and a benchmark point of 
the MSSM parameter space, Ref. [15] proved that the impact of the inclusion of supersymmetric decays on the mass exclusion limits is about $200-300 \mathrm{GeV}$.

\section{Bilepton signature at the LHC}

\subsection{Theoretical framework}

The gauge structure of the bilepton model [8], also known as 331 model, is $S U(3)_{c} \times S U(3)_{L} \times$ $U(1)_{X}$. It is a quite appealing framework, as it treats the third quark family in a different way with respect to the other two families and predicts anomaly cancellation between families, and not for each generation, as long as the numbers of colours and families are equal. As for quarks, three exotic states are predicted: $D$ and $S$, having charge $-4 / 3$ and lepton number $L=+2$, and $T$, with charge $5 / 3$ and $L=-2$. Moreover, the model accommodates four neutral Higgs bosons, three scalar $\left(h_{1}, h_{2}\right.$ and $\left.h_{3}\right)$ and one pseudoscalar $\left(h_{1}\right)$, along with two singly-charged $\left(h_{1}^{ \pm}, h_{2}^{ \pm}\right)$and one doubly-charged $\left(h_{1}^{ \pm \pm}\right)$Higgses. In the vector sector, a $Z^{\prime}$ is also predicted, as well as singly- and doubly-charged bileptons $Y^{ \pm}$and $Y^{ \pm \pm}$, with $L=2\left(Y^{++}\right.$and $\left.Y^{+}\right)$or $L=-2\left(Y^{--}\right.$and $\left.Y^{-}\right)$.

\subsection{Phenomenology of bileptons at the LHC}

Production of $Y^{++} Y^{--}$pairs in Drell-Yan processes were investigated in [16] and an exclusion limit about $m_{Y^{ \pm \pm}}>850 \mathrm{GeV}$ was set, after comparing the bilepton prediction with the ATLAS search for doubly-charged Higgs bosons [9]. In this talk, I will instead review the main results in [17], where the authors explored bilepton pairs accompanied by two jets:

$$
p p \rightarrow Y^{++} Y^{--} j j \rightarrow\left(\ell^{+} \ell^{+}\right)\left(\ell^{-} \ell^{-}\right) j j .
$$

The analysis in [17] features a benchmark point where the bileptons $Y^{ \pm \pm}$have mass about 873 $\mathrm{GeV}$, the $Z^{\prime}$ is leptophobic and with $m_{Z^{\prime}} \simeq 3 \mathrm{TeV}$, the exotic quarks are at $1.6-1.7 \mathrm{TeV}$ and the new Higgs bosons are too heavy to contribute to bilepton phenomenology. In the reference point of [17], the LO cross section of process (3.1), calculated by MadGraph, reads, at $\sqrt{s}=13 \mathrm{TeV}$ : $\sigma\left(p p \rightarrow Y^{++} Y^{--} j j \rightarrow 4 \ell j j\right) \simeq 3.7 \mathrm{fb}$. Moreover, the following acceptance cuts are imposed: $p_{T, j}>30 \mathrm{GeV}, p_{T, \ell}>20 \mathrm{GeV},\left|\eta_{j}\right|<4.5,\left|\eta_{\ell}\right|<2.5, \Delta R_{j j}>0.4, \Delta R_{\ell \ell}>0.1, \Delta R_{j \ell}>0.4$.

In Fig. 2 (left) I present the invariant-mass spectrum of same-sign lepton pairs (left) in our signal (solid histogram) and in the background processes $Z Z j j$ (dashes) and $t \bar{t} Z$ (dots), according to MadGraph+HERWIG; in the right panel of Fig. 2, $\theta_{\ell \ell}$ is instead the angle between same-sign leptons in signal and background processes. From Fig. 2 one learns that our bilepton signal can be easily separated from the backgrounds. The invariant mass $m_{\ell \ell}$ distributions are indeed very different: the signal peaks at $m_{Y^{++}} \simeq 873 \mathrm{GeV}$ and manifests as a narrow resonance, whereas the backgrounds yield broader spectra, peaked around $80 \mathrm{GeV}$ and vanishing for $m_{\ell \ell}>350 \mathrm{GeV}$. Regarding the angular distributions, our signal predicts a higher event fraction at $1<\theta_{\ell \ell}<2$ with respect to all backgrounds.

\section{Conclusions}

I investigated two scenarios featuring heavy vectors at the LHC, which have not yet received adequate consideration from the experimental collaborations, namely supersymmetric decays of 

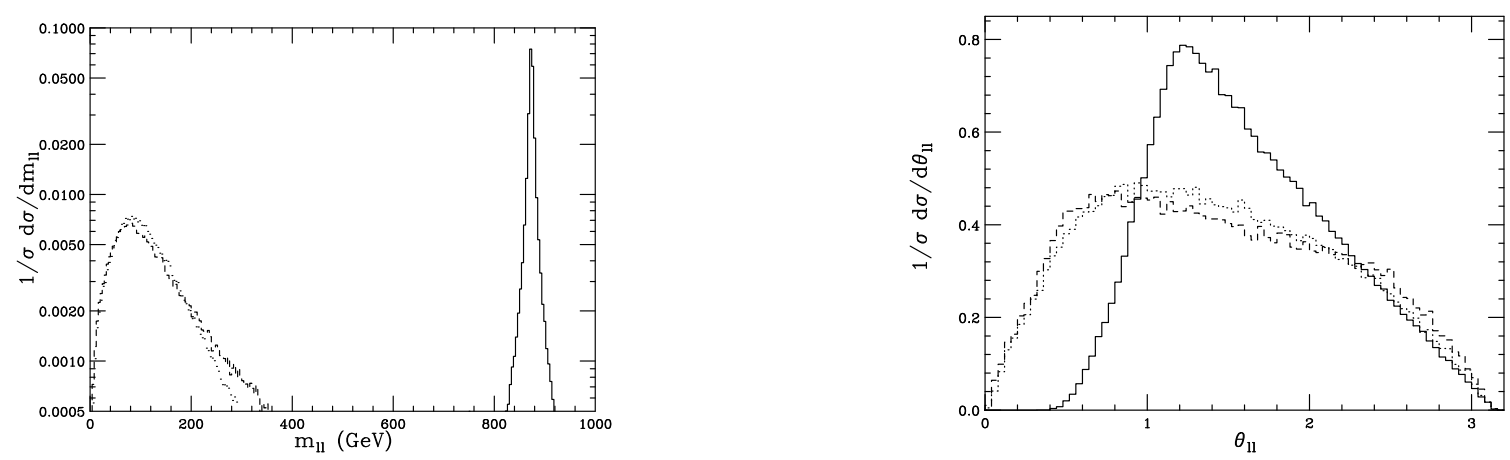

Figure 2: Left: invariant-mass distribution of same-sign lepton pairs. Right: opening angle between samesign leptons. Plotted are the spectra yielded by our signal (solid), $Z Z j j$ (dashes) and $t \bar{t} Z$ (dots) backgrounds.

GUT-inspired $Z^{\prime}$ bosons and doubly-charged vector bilepton production. I demonstrated that primary $Z^{\prime}$ decays into charginos lead to final states with charged leptons and missing energy, which can be discriminated from direct non-resonant chargino production. Likewise, the production of vector-bilepton pairs and two jets can yield a visible signal at the LHC, which can be separated from the backgrounds setting typical acceptance cuts. It is now certainly worthwhile reconsidering the investigation in [16] on vector $Y^{++} Y^{--}$production in Drell-Yan processes, so as to understand how the final-state distributions fare with respect to scalar $\mathrm{H}^{++} \mathrm{H}^{--}$production, studied in $[9,10]$, and used in [16] to set the exclusion limits on the $Y^{ \pm \pm}$mass. This is in progress.

\section{References}

[1] P. Langacker, Rev. Mod. Phys. 81 (2009) 1199.

[2] ATLAS Collaboration, arXiv:1707.02424 [hep-ex].

[3] CMS Collaboration, CMS-PAS-EXO-16-031.

[4] ATLAS Collaboration, Phys. Rev. D96 (2017) 052004.

[5] CMS Collaboration, CMS-PAS-EXO-16-056.

[6] G. Corcella and S. Gentile, Nucl. Phys. B866 (2013) 293; Erratum-ibid. B868 (2013) 554.

[7] G. Corcella, Eur. Phys. J. C75 (2015) 264.

[8] P.H. Frampton, Phys. Rev. Lett. 692889 (1992).

[9] ATLAS Collaboration, ATLAS-CONF-2017-053.

[10] CMS Collaboration, CMS-PAS-HIG-16-036.

[11] F. Staub, Comput. Phys. Commun. 184 (2013) 1792.

[12] W. Porod and F. Staub, Comput. Phys. Commun. 183 (2012) 2458.

[13] J. Alwall et al., JHEP 1407 (2014) 079.

[14] G. Corcella et al., JHEP 0101 (2001) 010.

[15] G. Corcella, EPJ Web Conf. 60 (2013) 18011.

[16] A.A. Nepomuceno, F.L. Eccard and B. Meirose, Phys. Rev. D94 055020 (2016).

[17] G. Corcella, C. Corianò, A. Costantini and P.H. Frampton, Phys. Lett. B773 (2017) 544. 